\title{
STUDY ON THE RELIABILITY OF PLUGGING AND DETACHING FISHEYE CONTACT
}

\author{
Maohua Xiao', Jun Guo ${ }^{2}$, Xueyong Chen ${ }^{3}$, Meng Xu', Meng Chen ${ }^{5}$, Xiao Zhao \\ 1,4,5 College of Engineering, Nanjing Agricultural University, Nanjing 210031, China \\ 1,2,6 Faculty of Engineering and the Environment, University of Southampton, SO17 1BJ, UK \\ ${ }^{3}$ China Aviation Optoelectronics Technology Co., Ltd, Luo Yang, 471003, China \\ xiaomaohua@njau.edu.cn
}

\begin{abstract}
Aiming at contact instability and the deformation problem of possible contact between the fisheye structure and the through hole in the fisheye shaped contact pairs, for pair alignment of fisheye shaped pairs, simulation analysis and experimental research of plug and play is conducted in this paper. Firstly, the important parameters of each structure of fisheye shaped contact pairs are analyzed, then, according to analyze the influence factors of fisheye shaped contact on deformation and insertion force, and based on ABAQUS software to study plug and simulate fisheye shaped contact under different parameters, it is concluded that the insertion force, structural deformation and signal transmission of fisheye shaped contact have great influence on the contact force of fisheye under different material parameters and structural parameters. Multiple plug experiment on fisheye shaped contact pairs was conducted, and the reliability of plug and socket contact insertion has significant influence on plug and play reliability was obtained, thus theoretical basis for the design of fisheye shaped structure, the setting of tolerance range and the control of frequency of use was provided.
\end{abstract}

Keywords: Fisheye contact pair; Insertion and withdrawal force; Structural deformation; Simulation analysis; ABAQUS

\section{Introduction}

The contact is the signal transmission of the electronic equipment in the electrical connector in many electrical connections, and the electrical connector transfers the signal from the input of the electrical connector to the output terminal, therefore, the reliability of its contact directly affects the transmission of signals ${ }^{[1]}$. Fisheye contact is one of the important parts of an electrical connection, which high precision, high density, high reliability and miniaturization features make it widely used in industrial control, digital electronic products, aviation, navigation, instruments and meters and other fields. At present, the leading global companies in the field of interconnection products can be divided into Tyco, Amphenol and Molex, etc. They are the leading companies in the industry. They started relatively earlier and have many connector series covering a wide range of areas ${ }^{[2]}$. The connection performance of electrical connectors is easily affected by external vibration or temperature changes, and field data are demonstrated that 30\% to $60 \%$ of electrical connectors have connection reliability problems ${ }^{[3]}$. For distinct signal transmission devices for application of contacts, there have been Chen Wenhua ${ }^{[4]}$, and Yang Fenwei ${ }^{[5]}$ exploring the failure mechanism and reliability of docking contact, however, there has been no failure to rank classification[6]. Besides the failure rate of interconnection mechanism is very important.

Through analysis of failure rate of American aviation equipment, the failure of electronic components in equipment is about $40 \%$, and there are $20 \%$ to $30 \%$ of the failure of connectors ${ }^{[7]}$.At present, domestic enterprises are taking basic technology learning as an essential aspect, and integrate the basic technology into the development of new products. On the field of contact reliability of connectors, experts have tested the total resistance model, key factors, failure forms and reliability ${ }^{[8]}$ to ensure that the product meets performance requirements ${ }^{[9]}$, but the performance of fisheye shaped contact pair is very little, so it is necessary to study fisheye contact pair.

In this paper, fisheye contact pair are taken as the object of study. The material parameters and structural parameters are studied respectively, and the influence of the change of critical parameters on the deformation of fisheye structure is also studied. Combined with ABAQUS and MATLAB software, the pull in force of fisheye contact in different fisheye positions is analyzed, the process of testing multiple plug insertion force determined by controlling the deformation, fisheye and pore structure to reduce 
contact failure probability, improve the utilization rate of contact, and provide a theoretical basis for the practical application of the fisheye contact.

\section{Structure Analysis of Fisheye Contact Pair}

\subsection{Analysis of fisheye structure}

Fisheye structure is the key contact between high speed connector and printed circuit board, and that is also known as the eye of a needle in the academic structure. The design and implementation of a experiment [10] system for signal integrity problem research on the transmission rate over the speed of the connector. In applications, board level connectors are pressed through the fisheye structure into the via of the printed board and the fisheye shaped structure is deformed by extrusion so as to generate a certain insertion force. The object of this study is fisheye structure of contact, the fisheye structure and the cross hole of the backboard.

The structure is shown in Fig. 1 and the key structural dimension parameters are shown in Fig. 2. The drilled hole is $0.55 \mathrm{~mm}$, and the finished hole is $0.45 \mathrm{~mm}$. In the simulation, the fisheye shaped structure is established according to the actual situation. The system takes into account the influence of position offset on the process of insertion and the structure of fisheye. Pins and sockets are essential for electrical signal transfer and functional control of electrical connectors.

The key part of fisheye structure size as shown in Fig. 2 the "※" parameter is shown, changes in these parameters will have great influence on the structure of fisheye and through-hole plug. The change of these dimension parameters has a great influence on the structure of fisheye and the insertion force of through hole.

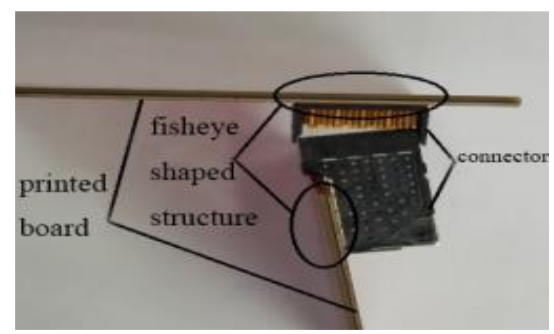

(a) Connector socket on the application of the fisheye structure

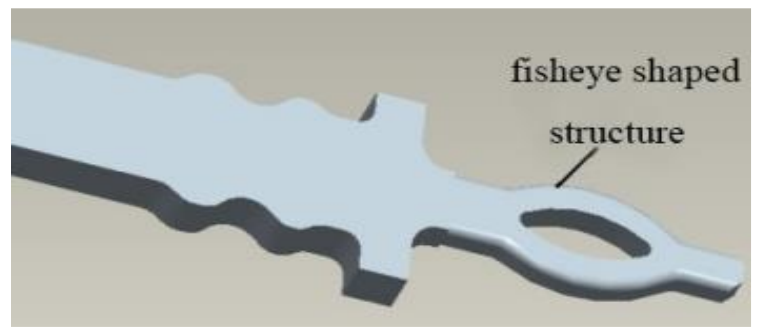

(b) The back of the hole

Figure 1: Fisheye structure application

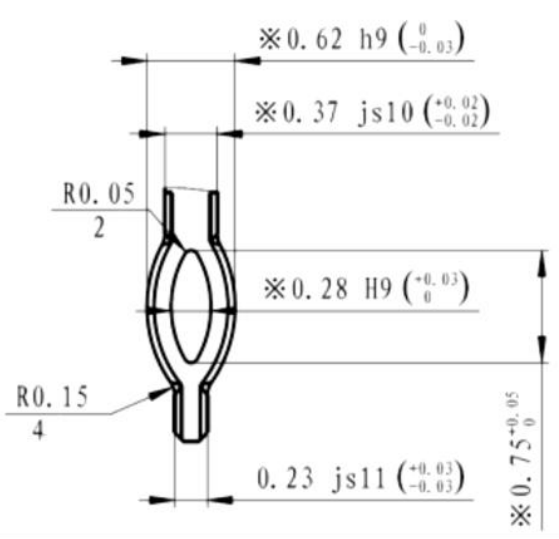

Figure2: Fisheye structure parameters

The key mechanical performance is involved in fisheye shaped contact is plug force. Insertion and withdrawal are a major performance indicator for connector assembly. It is not only related to the degree of ease of assembly, but also to the reliability of the whole system after assembly. The insertion force of the insertion process causes assembly difficulties, and at the same time, it will affect the insertion loss[11]. Withdrawal force is reduced, and the smaller withdrawal force will cause the connection to be done unreliable in the cabinet, which will seriously cause the signal to break.

\subsection{Analysis of cross hole structure}

The structure of the PCB is shown in Figure 3. The structural parameters included are mainly drilled holes and finished holes[12-13]. There is a copper deposit of about 0.05 between the drilled hole and the finished hole. When the fisheye structure and the whole are assembled, the plastic deformation of the sinking copper will have a certain influence on the insertion force.

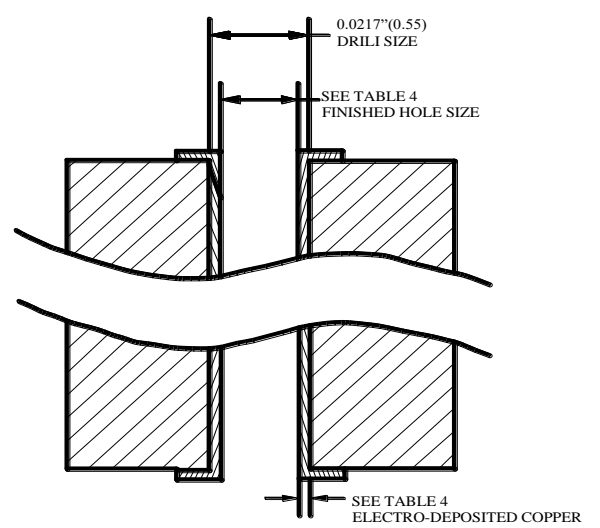

Figure 3: Sketch map of through hole structure 


\section{The Study on Different Parameters}

\subsection{Analysis of the influence of material parameter change on insertion force}

Fisheye contacts with different material parameters have very different sensitivity to the insertion states[14]. According to data transmission efficiency of fisheye structure, the fisheye structure is often made of different materials, such as C7025, C18080, C18140, C18070 and so on. The insertion force curves of the through hole and the fisheye structure are changed when the material parameters are different. When the fisheye shaped structure is the same, different insertion parameters can be obtained with different material parameters, and C18140 materials and C18070 curve of coincidence degree is relatively large, two materials arrive at almost the same time kurtosis value; C7025 material force kurtosis value is the highest. From the analysis of curve change and plug force, it is found that the insertion force and withdrawal force of the through hole and fisheye shaped structure are larger when C7025 material is utilized[15-16]. According to the material parameters, there is little difference between the elastic modulus of several materials, so the size of the plug force is mainly controlled by yield strength. The yield strength is the yield limit for the yielding of metal materials, that is, the resistance to a small amount of plastic deformation. The yield strength of the material is determined mainly by the properties of the material itself, and by the influence of the heat treatment process. For a metallic material without apparent yield, the stress value that produces $0.2 \%$ residual deformation is defined as its yield limit, referred to as the conditional yield limit or the yield strength. If the external force greater than this limit, it will cause the part to fail permanently and will not recover. When the external force is greater than this limit, the parts will be permanently deformed, and the parts will be restored to their original state. This is because when the yield strength of the material is lower, the through hole and the fisheye shaped structure are prone to yield deformation, resulting in smaller insertion force.

\section{Analysis of the Influence of the Change of Parameters on the Insertion Force}

\subsection{The influence of the width of the outer edge on the insertion force}

Rim width is an important parameter of fisheye structure, strict control of the parameters in the design, and the width of the edge model is $0.62(0,-$ 0.03 ), analysis of the influence of the width of the outer withdrawal for the system in the simulation, so the analysis of the size were $0.57,0.59,0.62,0.64$ the plug size, specifically through the results shown in Fig. 4 and Fig. 5.

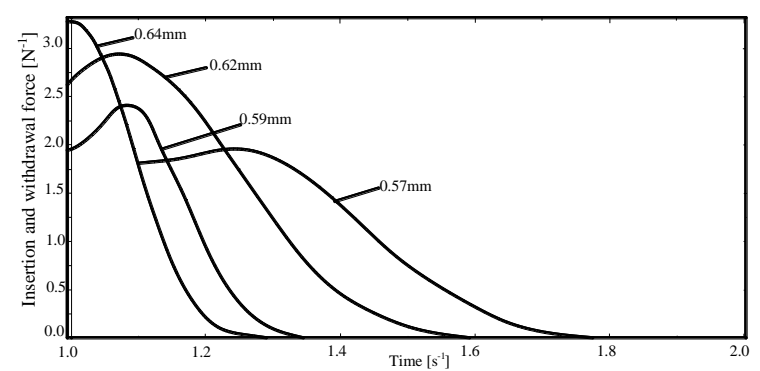

Figure 4: Insertion force curves

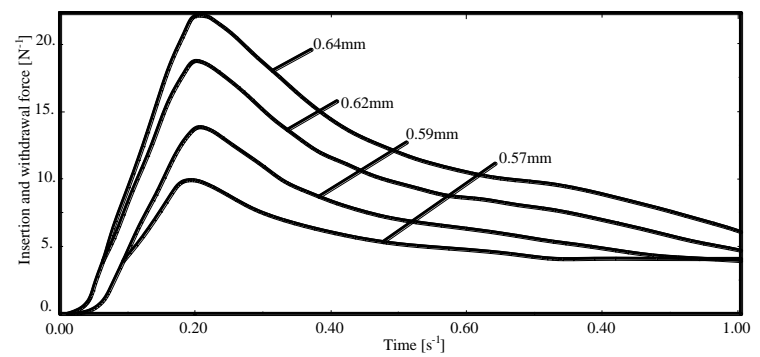

Figure 5: Comparison of withdrawal curves

Fig. 4 and Fig. 5 show the withdrawal force curves at different outer width. The figure is shown that both the insertion force and the withdrawal force increase as the width of the outer edge increases. the withdrawal stage and the width of the outer edge is larger. The withdrawal force will soon become smaller. This is because the width of the outer edge is larger. The plastic deformation of the fisheye structure is obvious, and the rebound amount of the material is limited, which makes the withdrawal force decrease rapidly. With the increase of the width of the outer edge, both the insertion force and the withdrawal force showed an increasing trend. the withdrawal stage and the width of the outer edge is larger. The withdrawal force will soon become smaller. This is because the greater the width of the outer edge, the more noticeable the plastic deformation of the fisheye structure, and the rebound amount of the material is limited, resulting in rapid reduction of withdrawal force. The change tendency of insertion force is different under different size parameters. With the increase of outer edge width, the insertion force increases rapidly, and the increase amplitude is obvious compared with the withdrawal force. This is because the thickness of the two sides of the fish eye increases with the increase of the width of the outer edge, while the interference increases with the contact with the hole, resulting in an increase in the insertion force.

The results show that the insertion force increases rapidly with the increase of the width of the outer edge, compared with the withdrawal force. The increase is obvious. This is because the thickness of the two sides of the fisheye structure increases with the increase of the width of the outer edge, 
while the interference increases with the contact with the hole, resulting in an increase in the insertion force.

\subsection{The influence of the width and length of the hole on the insertion and withdrawal force}

In the simulation, the influence of the inner hole width of the insertion force is analyzed, and the width of the hole is $0.28(+0.03,0)$ in the model. Therefore, the size of the plug force at $0.26,0.28$, 0.31 and 0.33 is analyzed. With the increase of the inner hole width, the insertion force shows a decreasing trend, and the tendency of decrease is close to the pull-out force. This is explained by the fact that when the width of the inner hole increases, the structural thickness of both sides of the fish eye decreases, and the fisheye structure is more prone to deformation.

In this model, the hole width is $0.75(+0.05,0)$, in the simulation system for the analysis of inner hole length of plug, so the analysis of the size were 0.73 , $0.75,0.80,0.82$ of the plug size according to the change trend of force from different hole length, when the length of the inner hole the larger, the pressing force becomes smaller, but to a lesser extent reduced. Report to the pull-out force, the decrease trend is relatively close.

\subsection{The influence of root width of fisheye on insertion force}

In this model, the width of the hole is $0.37(+0.02$, $-0.02)$, and the influence of the width of the root of fisheye structure of the insertion force is analyzed. The size of the plug force at $0.73,0.75,0.80$ and 0.82 is analyzed.

According to the changing trend of the insertion force of different fish root width, the insertion force decreases with the increase of the width of the fish eye root, and the trend is obvious when compared with the pull-out force. Therefore, in the eyes of the given fish size parameters, can help to reduce the fisheye eye before the $0.37(+0.02,-0.02) 0.36$ $(+0.05,0)$, optimized for optimized insertion force, the pull-out force is basically not affected. At the same time, the enlargement of tolerance range is useful to reduce the machining difficulty and improve the machining efficiency.

\section{Analysis of the Influence of Eye Hole Deviation on Plug Force}

In fish processing, fisheye shaped holes inevitably exist in the wrong situation, therefore, combined with the actual situation in the simulation, ABAQUS simulation analysis of inner hole along the lateral offset of fisheye $0.03 \mathrm{~mm}$ and $0.06 \mathrm{~mm}$ and $0.03 \mathrm{~mm}$, down hole fisheye offset based on $0.09 \mathrm{~mm}$ and $0.06 \mathrm{~mm}$.

Fig. 6 is a fisheye shaped hole respectively along the lateral offset of $0 \mathrm{~mm}, 0.03 \mathrm{~mm}, 0.06 \mathrm{~mm}$, fisheye deformation state, when the eye hole not shifted, deformation of inner hole on both sides of the fisheye structure symmetry state; when the eye along the side of the inner hole offset, fisheye asymmetric deformation.

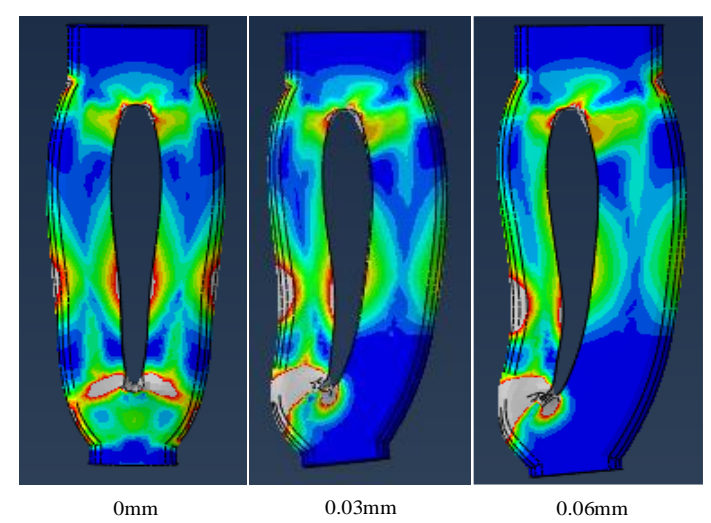

Figure 6: Lateral deformation of fisheye hole when $0 \mathrm{~mm}, 0.03 \mathrm{~mm}$ and $0.06 \mathrm{~mm}$ are plastically deformed

As showed in Fig. 6, the gray part is a serious plastic deformation area. When the fish eye hole is compensated for to one side, the plastic deformation at the offset side will become larger, and the risk of structural fracture will be increased. Therefore, the unilateral offset dimension of the fisheye inner hole structure should be strictly controlled by the application. Fig. 7 is the hole after the insertion force fisheye offset curve comparison, from the figure, the hole structure in offset fisheye after insertion and withdraw force decreased, this is because the fisheye hole offset, structure deformation occurred mainly in the area of eye side, compared with those at the shift situation it is easy to deform, resulting in reduced insertion and withdraw force.

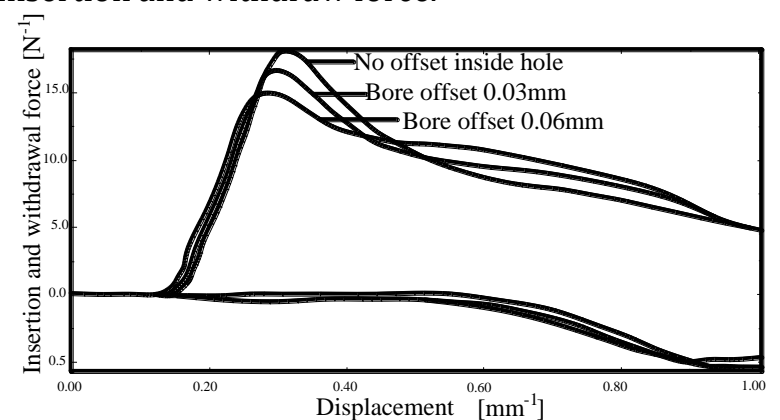

Figure 7: Withdrawal curve of side hole of fish eye hole

Fig. 8 is the comparison of the fisheye shaped plastic deformation at the same time when the fisheye hole is moved down. The grey part is a region with serious plastic deformation. It is shown from the figure that the area of plastic deformation of fish eye structure changes obviously with the increase of 
fisheye displacement. When the offset is 0 , the fisheye plastic deformation areas are mainly concentrated in the bottom of the fisheye holes, with the increase of the amount of downward, the fisheye plastic deformation region gradually shifted to the upper end of the fisheye holes, holes show that with fisheye down, lower fisheye severe structural force parts by fisheye holes transfer to the upper end of the inner hole of the fisheye.

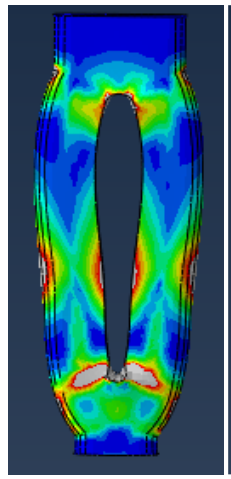

$0 \mathrm{~mm}$

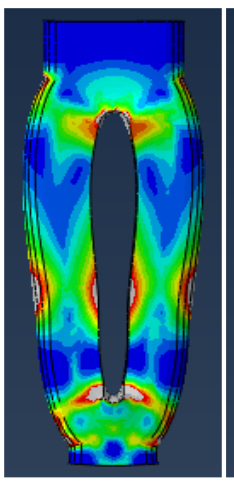

$0.03 \mathrm{~mm}$

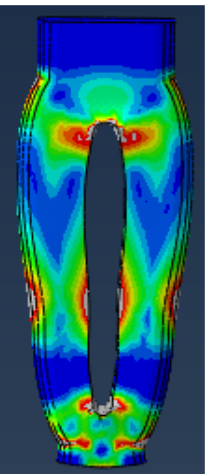

$0.06 \mathrm{~mm}$

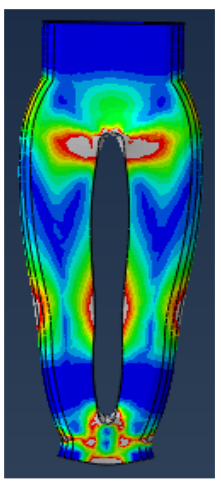

$0.09 \mathrm{~mm}$

Figure 8: Comparison of plastic deformation between fisheye shaped hole, 0 $\mathrm{mm}, 0.03 \mathrm{~mm}, 0.06 \mathrm{~mm}$ and $0.09 \mathrm{~mm}$

According to the practical situation, the upper hole is provided to keep the eye area, according to the design requirements, the fisheye shaped structures should be as large as possible retention by fisheye bore down, can change the structure of fisheye appropriate to keep regional state, increase the retention of fisheye.

Fig. 9 shows that the insertion force decreases and the withdrawal force increases as the displacement of the fisheye decreases as the displacement of the inner hole increases. According to the design requirements, the mechanical performance of fisheye can be improved after the fisheye inner hole is moved down.

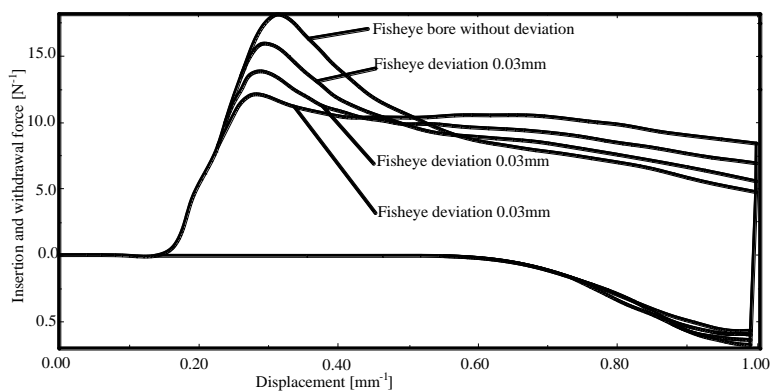

Figure 9: The influence of different offset on insertion force

When the fisheye hole is biased downward to $0.09 \mathrm{~mm}$, the insertion force is reduced from $18.6 \mathrm{~N}$ to $5.6 \mathrm{~N}$, which reduces by about $34.9 \%$, and the pulling force increases from $5.6 \mathrm{~N}$ to $6.8 \mathrm{~N}$, and increases by about $21.4 \%$. Therefore, in the design of fisheye structure, a certain amount of structure can be designed according to the inner hole of the fisheye.

When the displacement is large, the plastic deformation of the upper end of the eye hole is increased.

\section{Influence of Fisheye Shaped Contact on Cross Hole State and Insertion Force During Multiple Insertion}

In order to reduce the cost, printed boards usually require repeated use in order to ensure that the performance requirements of the product meet the requirements. If some manufacturers stipulated that the use of Chinese and Indian board to meet the needs of three times repeated plug. Therefore, the interpolation time of the same cross hole and multiple fisheye is simulated.

After the comparison between multiple plug views in the left picture in Fig. 10 for the first time on the plug hole and the eye of state, on the right picture for visas and fisheye fifth times to insert after the state, from the figure, after the first plug, the deformation of large orifice fish-eye. But after four time of insertion and removal, the deformation of the fish eye is basically the same, but only a slight change in the stress distribution. The earliest fish-eye and the fifth fisheye are the same as the through hole.

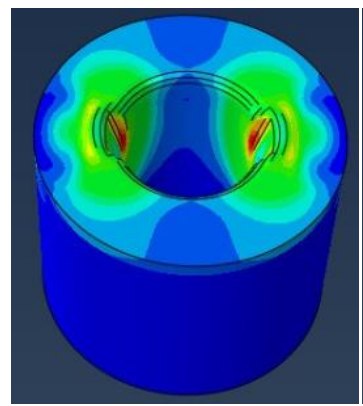

The first plug

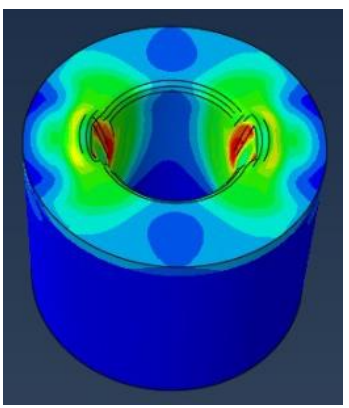

The last plug
Figure 10: After the first plug and last plug after the contrast chart 
Fig. 11 shows the force curve of the cross hole and the fisheye when they are inserted several times.

It is well known from the diagram that the insertion force curve at the time of insertion is different from the subsequent insertion force curve, but there is no difference in withdrawal force. This is explained by the fact that, for the first time, a plastic deformation has occurred on the inserted through hole, so that the contact between the second fisheye and the cross hole is required to travel longer distances to make contact with the through hole.

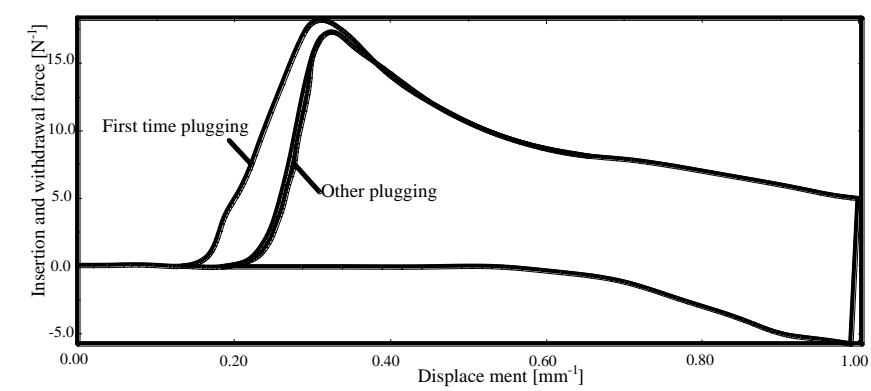

Figure 11: Comparison of the first to fifth withdrawal force curves

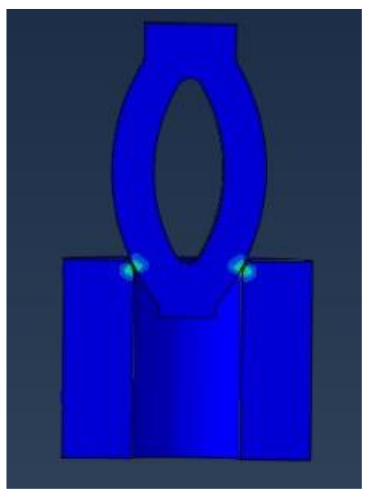

The first plug states

Figure 12: Comparison of the insertion states

At the same time, the position and direction of the force of the fish eye have changed after the deformation of the hole at the cross hole, as showed in Fig. 12. Therefore, the insertion force of the fish eye is distinguished obviously. When the plug is put in place, the fisheye has an overall deformation.

When the plug is inserted, the fisheye structure has little change in the force mode, so the difference of the pulling force is small.

The simulation results demonstrate that the cross hole and the first fisheye pair affect the subsequent interpolation process directly. And many fisheye after many times to insert, still can guarantee enough to keep the force, but many times the plug will affect the whole coating, surface quality and other factors.

\section{Conclusion}

(1) When the fisheye shaped structure adopts different material parameters, the insertion force of dissimilar materials is different. The difference between the inserted forces is obvious, because the elastic modulus of the selected material is basically the same, so the core material parameter affecting the insertion force is the yield strength, and the yield strength is inversely related to the insertion force.

(2) The structural parameters of the width of fisheye shaped structure, the width and length of the inner hole have great influence on the insertion force, and the insertion force is positively related to the width of the outer edge of the fish eye, and inversely related to the width and length of the inner hole.

(3) According to the simulation results, the control side offset within $0.02 \mathrm{~mm}$, the control bore down amount of fisheye in the range of $0.06 \mathrm{~mm}-0.1 \mathrm{~mm}$, can ensure the fisheye structure with higher pullout force and fisheye root structure plastic deformation controlled, fracture risk is small.

(4) fisheye shaped contact will cause the deformation of the fisheye shaped structure and the through hole structure, which will lead to the change of the insertion force. Therefore, the number of insertions and withdrawal should be controlled in practical applications.

\section{References}

[1] K. Miyanaga, Y. Kayano, H. Inoue. A Study on Lifetime and Reliability of Asymmetric Electrical Contact Pair[J]. Ieice Technical Report Emd, 110(2010), No. 4, 11-16.

[2] Y. W. Park, H. G. Joo, K. Y. Lee. Effect of intermittent fretting on corrosion behavior in electrical contact. Wear[J]. 268(2010), No. 3, 353360.

[3] W. B. Ren, P. Wang, Y. H. Fu, C. F. Pan, J. Song. Effects of temperature on fretting corrosion behaviors of gold-plated copper alloy electrical contacts, Tribology International[J]. 83(2015), No. 1, 1-11.

[4] W. H. Chen, J. Liu and L. Gao. Accelerated Degradation Reliability Modeling and Test Data Statistical Analysis of Aerospace Electrical Connector. Chinese Journal of Mechanical Engineering[J]. 24(2011), No. 6, 957-962. 
[5] F. W. Yang. Electrical Connector Destructive Physical Analysis (DPA) Discussion. Quality and reliability[J]. 04(2017), No. 4, 32-37.

[6]Mikolajczak et al. Subminiature electrical connector including over-voltage and overcurrent circuit protection. United States Patent[P]. US 7,497,737 B2,Mar. 3, 2009

[7] W.H. Chen, X.Z. Kong, P. Qian, M. Gao. and Z.P. Zhu. Influence of plug and play on performance degradation of stored electrical connector. Mechatronics Engineering[J]. 05(2016), No. 3, 508-513.

[8] A. Zambrano, J. M. D. Silva. Signal Integrity and Interconnections Test on Technical Fabrics// IEEE, International Mixed-Signal, Sensors, and Systems Test Workshop. IEEE Computer Society[J]. 03(2012), No.2, 79-84.

[9] B. Huang, X. B. Li, Z. Zeng, Y. H. Zhang. An Approach to Optimize the Consistency of Insertion and Extraction Forces of Contacts Based on Robust Design Principle. Chinese Journal of Mechanical Engineering[J]. 53(2017), No. 4, 190197.

[10] J. Pan, F.J. Jin and W. H. Chen. Structural analysis and plug and socket test of electrical connector. China Mechanical Engineering[J]. 24(2013), No. 12, 1197-1200.

[11]Anbin Ren, Li Cui, Jinbao Chen. Fretting Behavior of $\mathrm{Au}$ Plated Copper Contacts Induced by High Frequency Vibration[C]. Electrical Contacts (Holm), 2012 IEEE 58th Holm Conference on 2326 September. 2012.
[12] A.R Reber and B.J. Nin. Experimental and analytical studies of the connector insertion phase. IEEE Transactions on Components and Packaging Technologies[J]. 31(2008), No. 4, 751757.

[13] Xiaomei. Xu, Yiping Jiang and Heow Pueh Lee. Multi-objective optimal design of sandwich panels using a genetic algorithm. Engineering Optimization [J]. 49(2017). No. 10,1665-1684

[14] X.Y. Guan, N.Q. Shu and B. Kang: Multiphysics analysis of plug-in connector under steady and short circuit conditions. IEEE Transactions on Components, Packaging and Manufacturing Technology[J].05(2015), No. 3, 320-327.

[15] S. Ling. Optimization Method of Quality Consistency for Insertion Force of Electrical Contact Based on Robust Design Principle. Journal of Mechanical Engineering[J].53(2017), No. $4,190$.

[16] A. E. Manfalouti, N. B. Jemaa, R. E. Abdi. A New Experimental Method for the Determination of Connector Parameters in Insertion and Extraction Phase. IEICE TRANSACTIONS on Electronics[J]. 08(2004), No. 3, 1289-1294.

\section{Acknowledgments}

The research is funded partially by the students scientific research innovation training project of Nanjing Agricultural University(1730A08) and the Fundamental Research Funds for the Central Universities fund (KYZ201760).

Corresponding Author: Maohua Xiao, E-mail: xiaomaohua@njau.edu.cn 